\title{
BMJ Open Prevalence and distribution of HPV infection and subtypes in oral squamous cell carcinoma in Africa: a systematic review protocol
}

\author{
Haly Karen Holmes (D) ,' Amir Afrogeh,, ${ }^{2,3}$ Henry Adeola, ${ }^{1,4}$ \\ Faheema Kimmie-Dhansay, ${ }^{1}$ Mark E Engel ${ }^{5}$
}

To cite: Holmes HK, Afrogeh A, Adeola $\mathrm{H}$, et al. Prevalence and distribution of HPV infection and subtypes in oral squamous cell carcinoma in Africa: a systematic review protocol. BMJ Open 2021;11:e049922. doi:10.1136/ bmjopen-2021-049922

- Prepublication history for this paper is available online. To view these files, please visit the journal online (http://dx.doi org/10.1136/bmjopen-2021049922).

Received 09 February 2021 Accepted 28 May 2021

Check for updates

(C) Author(s) (or their employer(s)) 2021. Re-use permitted under CC BY-NC. No commercial re-use. See rights and permissions. Published by BMJ.

${ }^{1}$ Dentistry, University of the Western Cape, Bellville, South Africa

${ }^{2}$ Oral Pathology, University of the Western Cape, Bellville, South Africa

${ }^{3}$ National Health Laboratory Service at Groote Schuur Hospital, Observatory, South

Africa

${ }^{4}$ Division of Dermatology, University of Cape Town Faculty of Health Sciences, Observatory, South Africa

${ }^{5}$ Medicine, University of Cape Town Faculty of Health Sciences, Observatory, South Africa

Correspondence to

Dr Haly Karen Holmes;

hholmes@uwc.ac.za

\section{ABSTRACT}

Introduction Human papillomavirus (HPV) is an established risk factor for oropharyngeal squamous cell carcinoma, regardless of a history of other known risk factors such as alcohol and tobacco. While cases of HPV-related oral squamous cell carcinoma (OSCC) are increasing in the USA, Europe and South Central Asian countries, little is known about the impact of the disease on the African continent.

Methods and analysis We describe a protocol for a systematic review to synthesise the best current evidence to assess the disease burden in Africa. Electronic databases including EBSCOhost, MEDLINE, CINAHL, ACADEMIC SEARCH COMPLETE, ScienceDirect, Web of Science, Scopus, SciCENTRAL, Cochrane Library, International Prospective Register of Systematic Reviews) and WorldCAT will be comprehensively searched for studies reporting on the defined outcomes, in Africa, published from 1985 (when HPV was first reported) to the latest current entries, with no language restriction. Supplemental handsearching of grey literature, conference abstract proceedings, reference lists of included studies and citations in Google Scholar will be conducted. Authors will be contacted, where necessary, to assist with missing data. A customised data extraction form, with specified criteria, will be used for data extraction. Overall study quality assessment will be done using an appropriate risk of bias tool suited to the study design. Where available, qualitative data from studies reporting on the outcomes will be captured on the data extraction form. Using Stata software, we will apply the random-effects meta-analysis model to aggregate prevalence estimates with $95 \% \mathrm{Cl}$, incorporating the Freeman-Tukey transformation to account for between-study variability. A narrative report of the findings will be presented where data are insufficient in terms of the outcome/s. Subgroup analysis will be done subject to sufficient available data.

Ethics and dissemination Ethics approval or written consent is not required as the review will be conducted using published data. The findings will be distributed through a peer-review publication and conference presentation.

\section{INTRODUCTION}

Human papillomavirus (HPV) is an established risk factor for oral squamous cell
Strengths and limitations of this study

- This study will present the best evidence on prevalence of human papillomavirus (HPV) OSCC in Africa.

- The results will have implications for policy, practice and further research and inform health and policy makers on current practices relative to accepted international standards.

- This review will rely on reporting within studies and may not represent the true prevalence of HPV oral squamous cell carcinoma (OSCC).

- The review findings may not be representative of the true population-based HPV OSCC on the African continent.

carcinoma (OSCC), particularly within the oropharyngeal region, ${ }^{12}$ regardless of a history of other known risk factors such as alcohol, tobacco or both. HPV head and neck cancer (HNC) represents a distinct disease entity compared with HPV negative tumours. Patients with HPV+tumours present at a younger age and are less likely to partake in excessive smoking and alcohol use. ${ }^{2-4}$ Although the annual incidence of smokingrelated head and neck squamous cell carcinoma's (HNSCC) has decreased significantly over the recent decade at a rate of $1,85 \%$ annually, HPV-associated HNSCC incidence has increased significantly at a rate $0.8 \%$ annually. ${ }^{3}$

\section{Context for this review}

HPV belongs to a family of viruses that is sexually transmitted. ${ }^{5}$ It is a double-stranded DNA virus, whose genome contains open reading frames which are potential coding sites for six early (E) proteins and two late (L) proteins, ${ }^{6}$ implicated in oncogenic infection by HPV. Various diagnostic techniques to detect HPV infection in OSCC have been used in studies. In 2018, the American Society of 
Clinical Oncology recommended that p16 immunohistocytochemistry positivity be used as a surrogate marker for High risk HPV expression, with at least $70 \%$ nuclear and cytoplasmic expression. $^{27}$

Globally, the exact prevalence and distribution of HPV in OSCC specifically is uncertain and needs to be determined as its incidence in $\mathrm{HNC}$ reportedly varies by geographical location. Cases of HPV-related OSCC are reportedly increasing in the USA, Europe and SouthCentral Asian countries, ${ }^{4}$ but little is known about the burden and impact of the disease on the African continent. ${ }^{8}$ In the African context, the proportion of patients with HPV-positive disease is hard to estimate, as many referral centres do not have access to routine testing. It has been hypothesised that the HPV pandemic will still emerge in developing countries owing to their burden of sexually transmitted infectious diseases. ${ }^{9}$

Oral cancer is among the top ten cancers by incidence and deaths in low-income or middle-income countries, as opposed to high-income countries, with an annual global incidence of $>650000$ and $>330000$ deaths cases. HNSCCs are the sixth most common cancer worldwide, approximately $50 \%$ of which occurs within the oral cavity. While the National Cancer Institute reports a marginal global decrease in the incidence of $\mathrm{HNC}$, whose primary risk factors include smoking and tobacco, HPV-associated HNC's have increased considerably since the debut isolation/discovery of HPV in a subset of HNC's (particularly oropharyngeal carcinomas).$^{10}$

The majority (90\%) of HNC's arise from the squamous epithelium lining of the oral cavity, larynx, pharynx and rarely nasal cavity. ${ }^{811}$ The oral cavity and oropharynx are separate regions that border each other but do not overlap. ${ }^{12}$ Oral SCC arise from the lips tongue (anterior two thirds of the tongue -anterior to circumvallate papillae), floor of mouth and hard palate and retromolar trigone oral cavity. The oral cavity anatomic sites include labial mucosa, buccal mucosa, floor of mouth, alveolar ridge and gingiva, anterior two-thirds of the tongue (anterior to circumvallate papillae). The oropharynx consists of the soft palate, base (posterior third of tongue), (palatine tonsil, palatoglossal folds, valleculae and posterior pharyngeal wall) and back of throat. These distinct anatomic borders separate the two sites from above the, the junction of the hard and soft palate, and from below, the circumvallate papillae. ${ }^{2} 12$ We propose to conduct a systematic review for the best evidence on the prevalence of HPV OSCC in adults in Africa.

\section{Objectives}

This protocol describes our intended systematic review to identify, appraise and synthesise best evidence to address the question: What is the prevalence and distribution of HPV subtypes in OSCC in Africa? The specific objectives/ secondary outcomes are (1) to determine the distribution of HPV-associated OSCC in Africa (by geographical site, oral cavity distribution, HPV subtype, demographic profile age and gender); (2) to describe the histological phenotype of HPV associated OSCC in Sub Saharan Africa; (3) to describe the molecular phenotype/signatures of HPV associated OSCC such as aneuploidy, TRAF3 loss, PIK3CA activating mutations, E2F1 amplifications) and (4) to describe the methods used to profile the HPV subtypes

\section{METHODS AND DESIGN}

The protocol for a systematic review on the prevalence of HPV OSCC is detailed in this article and has been submitted for registration in International Prospective Register of Systematic Reviews (PROSPERO). We will use specified inclusion and exclusion criteria to select studies for this systematic review, which will be reported according to Preferred Reporting Items for Systematic Reviews and Meta-Analyses guidelines Protocols. ${ }^{13}$ We will conduct a comprehensive literature search using the proposed search strategy and databases.

\section{Study eligibility criteria}

Inclusion criteria

Study design

We will include observational studies such as crosssectional, case-control and case series studies.

\section{Population/participants}

Participants will be all adults, older or equal to 18 years of age with OSCC, living in AFRICA, diagnosed from formalin-fixed paraffin-embedded specimens, confirmed by a pathologist and whose HPV status is confirmed by a standardised diagnostic test.

\section{Outcome}

The prevalence of HPV and subtypes in OSCC in Africa.

\section{Types of outcome measures}

1. To determine HPV prevalence by:

- Geographical distribution.

- Oral distribution.

- HPV subtype.

- Demographic profile (age and gender).

2. To determine the histological phenotype of HPV associated OSCC in sub-Saharan Africa.

3. To describe the molecular phenotype/signature of HPV associated OSCC such as aneuploidy, TRAF3 loss, PIK3CA activating mutations, E2Flamplifications.

4. Describe the methods used to profile HPV subtypes.

\section{Exclusion criteria}

We will exclude articles where full texts could not be obtained from the search, as were case reports, case series, qualitative measures/aspects and studies reporting children with HPV OPSCC.

\section{Search strategy for identification of studies}

We will use the CoCoPop framework (condition, context, population) for our search strategy. Studies must meet the inclusion criteria and report data pertaining to the 
numerator (number of HPV) and denominator (number of people with OSCC), which we will extract.

All relevant studies in Africa published from 1985 until date of searching, will be comprehensively searched and retrieved from the following databases: EBSCO Host, MEDLINE, ACADEMIC SEARCH COMPLETE, ScienceDirect, ERIC, WEB OF SCIENCE, SciELO), EMBASE, CENTRAL, COCHRANE LIBRARY, PROSPERO, SCOPUS).

Grey literature databases and other literature sources including conference abstract proceedings and NETD and WORLD CAT thesis databases will be searched. In addition, searches will be supplemented by handsearching reference lists of included studies and citations in Google Scholar, to identify other potential studies for consideration. Online registers will be searched for unpublished and ongoing studies. Study authors will be contacted where full text cannot be accessed or for any missing information.

A wide search strategy will be used to reduce publication bias, with no language restrictions. We will use varying combinations of text words and Medical subject headings (MeSH) terms used in PubMed/Medline to develop a preliminary search (box 1) and adapt the search strategy for every other database. Keywords proposed will be 'head and neck neoplasms (MeSH), 'mouth neoplasms' (MeSH)', 'HPV and human papillomavirus', 'papillomaviradae' (MeSH), 'Africa', prevalence. We will use the African countries filter was used, ${ }^{14}$ to ensure inclusion of all African countries in the search.

\section{Data collection and analysis}

Two reviewers will independently screen titles and abstracts identified through the literature searches for eligibility. Two authors will then access the full-text articles of the potential studies screened to compare the findings. Discrepancies will be resolved by discussion and consensus. Reasons for exclusion of any studies will be provided.

\section{Data extraction and management}

Search results will be managed using Rayyan systematic review web application. ${ }^{15}$ For each included article, two reviewers will independently extract descriptive and outcome data, using a data extraction form designed/ customised for that purpose. Any discrepancies will be resolved by discussion and consensus. Disagreements will be resolved by consensus or in consultation with a third reviewer. The data capturing sheet will include study characteristics such as author/s, country name, year of publication, national population, region (rural or urban), age range, sex, smoking status, study design, criteria for sample selection as well as sample material (paraffin-embedded) sample size, method of ascertainment of OSCC and HPV status, HPV subtype, diagnostic criteria used. The final data from included studies will be managed and analysed with Stata software version 15.0. Prevalence figures and 95\% CIs will be extracted
Box 1 Search Strategy used in MEDLINE : MeSH (Medical subject headings) and Keywords

("africa" (MeSH Terms]) OR "africa” [(All Fields]) OR “africa s" [(All Fields]) OR "africas" (All Fields]) OR "Comoros" [(All Fields]) OR "Djibouti" [(All Fields]) OR "Madagascar"[(All Fields]) OR "Malawi"[(All Fields]) OR "Seychelles"[(All Fields]) OR "Cameroon"[(All Fields]) OR "Central African Republic"[(All Fields]) OR "Chad"[(All Fields]) OR "Equatorial Guinea"[(All Fields]) OR ("gabon"[(MeSH Terms]) OR "gabon"[(All Fields])) OR "Morocco"[(All Fields]) OR "South Sudan"[(All Fields]) OR "Sudan"[(All Fields]) OR "Botswana"[(All Fields]) OR "Lesotho"[(All Fields]) OR "Swaziland"[(All Fields]) OR ("eswatini"[(MeSH Terms]) OR "eswatini"[(All Fields])) OR "Benin"[(All Fields]) OR "Burkina Faso"[(All Fields]) OR "Cape Verde"[(All Fields]) OR "Ghana"[(All Fields]) OR "Guinea"[(All Fields]) OR "Guinea-Bissau"[(All Fields]) OR "Mauritania" [(All Fields]) OR "Niger"[(All Fields]) OR "Senegal"[(All Fields]) OR "Sierra Leone"[(All Fields]) OR "Togo" ((All Fields]) OR ("burundi" [(MeSH Terms]) OR "burundi" [(All Fields])) OR ("eritrea"[(MeSH Terms]) OR "eritrea"[(All Fields])) OR ("ethiopia"[(MeSH Terms]) OR "ethiopia" [(All Fields]) OR "ethiopia s" [(All Fields])) OR ("kenya"[(MeSH Terms]) OR "kenya" (All Fields]) OR "kenya s"[(All Fields])) OR (“mozambique"[(MeSH Terms]) OR "mozambique"[(All Fields]) OR "mozambique s"[(All Fields])) OR ("rwanda"[(MeSH Terms]) OR "rwanda"[(All Fields]) OR "rwanda s"[(All Fields])) OR ("somalia"[(MeSH Terms]) OR "somalia" [(All Fields])) OR ("tanzania"[(MeSH Terms]) OR "tanzania" [(All Fields]) OR "tanzania s"[(All Fields])) OR ("uganda"[(MeSH Terms]) OR "uganda"[(All Fields]) OR "uganda s"[(All Fields])) OR ("zambia"[(MeSH Terms]) OR "zambia"[(All Fields]) OR "zambia s"[(All Fields])) OR ("zimbabwe"[(MeSH Terms]) OR "zimbabwe"[(All Fields]) OR "zimbabwe $\mathrm{s}$ "[(All Fields])) OR ("angola"[(MeSH Terms]) OR "angola"[(All Fields]) OR "angola s"[(All Fields])) OR ("algeria"[(MeSH Terms]) OR "algeria"[(All Fields])) OR ("egypt"[(MeSH Terms]) OR "egypt"[(All Fields]) OR "egypt $s$ "[(All Fields])) OR (“tunisia”[(MeSH Terms]) OR "tunisia”[(All Fields])) OR ("namibia"[(MeSH Terms]) OR "namibia"[(All Fields])) OR "South Africa"[(All Fields]) OR "the gambia"[(All Fields]) OR ("liberia"[(MeSH Terms]) OR "liberia"[(All Fields]) OR "liberia s"[(All Fields])) OR ("mali" [(MeSH Terms]) OR "mali”"(All Fields])) OR "Democratic Republic of Congo"[(All Fields]) OR "Ivory coast"[(All Fields]) OR ("libya"[(MeSH Terms]) OR "libya"[(All Fields])) OR ("mauritius"[(MeSH Terms]) OR "mauritius"[(All Fields])) OR ("nigeria"[(MeSH Terms]) OR "nigeria"[(All Fields]) $O R$ "nigeria s" [(All Fields])) OR "Sao Tome"[(All Fields]) OR "sub africa*"[(All Fields])) AND ("Papillomaviridae"[(MeSH Terms]) OR ("HPV" [Title/Abstract] OR "HUMAN PAPILLOMA VIRUS" [Title/Abstract] OR "HUMAN PAPILLOMAVIRUS"[Title/Abstract])) AND "Head and Neck Neoplasms"[(MeSH Terms]) AND ("PREVALENCE"[Title/Abstract] OR "INCIDENCE"[Title/Abstract] OR "PRESENCE" [Title/Abstract] OR "EPIDEMIOLOGY"[Title/Abstract])

or calculated from the available data. We will deal with missing data by contacting the corresponding authors.

\section{Risk of bias assessment for included studies}

Relevant risk of bias tools will be used according to the study design ${ }^{16}$ (table 1). Two independent reviewers will assess the methodological quality of the included literature for internal and external validity and general applicability of results. The adapted version ${ }^{17}$ of this 10 -item rating system will be used on domains specified within the inclusion criteria and outcomes (such as sampling frame and size, methods used to confirm diagnosis, outcomes and statistical reporting). Each item will be assigned a 


\begin{tabular}{ll}
\hline Table 1 Quality assessment tool & \\
\hline External validity & Points \\
\hline $1 \quad \begin{array}{l}\text { Was the study's target population a close } \\
\text { representation of the national population in } \\
\text { relation to relevant variables? }\end{array}$ \\
\hline $\begin{array}{l}\text { Was the sampling frame a true/close } \\
\text { representation of the target population? }\end{array}$ \\
$\begin{array}{l}\text { Was some form of random selection used } \\
\text { to select the sample? }\end{array}$ \\
$\begin{array}{l}\text { Was the likelihood of non-response bias } \\
\text { minimal }\end{array}$
\end{tabular}

Total points
Internal validity
$5 \quad$ Was data collected directly from patients
as opposed to proxy?
$6 \quad$ Was an acceptable case definition used in
the study?
$7 \quad \begin{aligned} & \text { Was the study instrument that measured } \\ & \text { the parameter of interest shown to have } \\ & \text { validity and reliability? }\end{aligned}$
$8 \quad \begin{aligned} & \text { Was the same mode of data collection } \\ & \text { used for all participants? }\end{aligned}$
$\begin{aligned} & \text { Was the length of the shortest prevalence } \\ & \text { period for the parameter of interest } \\ & \text { appropriate? }\end{aligned}$
$\begin{aligned} & \text { Were the numerators and denominators for } \\ & \text { the parameters of interest appropriate? }\end{aligned}$
Total points

score of 1 (yes) and 0 (no). The sum of all the score items from 0 to 10 will generate an overall quality score. Studies with scores of 8 or more, between 5-6 and less than 5, will be considered as low, medium and high risk, respectively (box 2).

\section{Strategy for data synthesis and analysis}

Where available, quantitative data from studies measuring and reporting on the outcomes will be captured on a data extraction form and a summary of the literature will be presented in data tables. Using Stata software, we will apply the random-effects meta-analysis model to aggregate prevalence estimates with $95 \% \mathrm{CI}$, incorporating the Freeman-Tukey transformation to account for betweenstudy variability. ${ }^{18}$

\section{Box 2 Key for measurement of risk of bias ${ }^{17}$}

Low (0-5 points).

Further research is very unlikely to change our confidence in the estimate.

Medium (6-8 points).

Further research is likely to have an important impact on our confidence in the estimate and may change the estimate.

High ( $>8$ points).

Further research is likely.
A narrative report of the results will be done where there are not enough studies measuring or reporting on the outcome/s. Subgroup analysis will be performed and stratified by secondary outcomes, provided there are sufficient data.

\section{Presenting and reporting of results}

Search results will be presented in the form of flow diagrams, to show the study selection and inclusion process. We will elaborate on the reason for excluded studies and provide a narrative description of outcomes for which there is insufficient data available for the desired outcome measures. Outcomes will be represented by Forest plots and tables where possible.

\section{Patient and public involvement}

There is no patient nor public involvement in the study.

\section{DISCUSSION}

\section{Anticipated significance of the study}

This systematic review will identify and present the available evidence for the prevalence of HPV OSCC on the African continent. The findings of this systematic review will have implications for policy, practice and research. It will inform / provide the status quo on burden of the disease burden on the African continent and diagnostic methods used for detection. The relevance of the findings on HPV prevalence on the African continent will inform health and policy-makers of the current practice relative to accepted international standards. We will discuss strengths and limitations of the study, highlighting any evidence gaps on the literature. The findings of this systematic review will be compared with that of other global systematic reviews, to compare the clinical picture of HPV OSCC in Africa.

Correction notice This article has been corrected since it was published. The acronym for oral squamous cell carcinoma has been corrected to OSCC.

Acknowledgements The authors would like to acknowledge the support of Prof Nicolette Roman and the Developing the Scholar Programme at UWC. No funding was received for this manuscript.

Contributors HKH conceptualised the review. HKH and MEE developed the design for the protocol and will be involved in data acquisition. HKH and MEE will analyse the data and HKH, MEE, AA, HA and FK-D will be involved in the interpretation of the results. All authors have consented to publication.

Funding The authors have not declared a specific grant for this research from any funding agency in the public, commercial or not-for-profit sectors.

\section{Competing interests None declared.}

Patient and public involvement Patients and/or the public were not involved in the design, or conduct, or reporting, or dissemination plans of this research.

Patient consent for publication Not required.

Provenance and peer review Not commissioned; externally peer reviewed.

Open access This is an open access article distributed in accordance with the Creative Commons Attribution Non Commercial (CC BY-NC 4.0) license, which permits others to distribute, remix, adapt, build upon this work non-commercially, and license their derivative works on different terms, provided the original work is properly cited, appropriate credit is given, any changes made indicated, and the use is non-commercial. See: http://creativecommons.org/licenses/by-nc/4.0/. 
ORCID iD

Haly Karen Holmes http://orcid.org/0000-0001-8297-8536

\section{REFERENCES}

1 Postma TC, Van Heerden WF. Is the human papillomavirus a mutual aetiological agent in oral and cervical squamous cell carcinoma? Anticancer Res 2003;23:3509-12.

2 Lewis JS, Beadle B, Bishop JA, et al. Human papillomavirus testing in head and neck carcinomas: guideline from the College of American pathologists. Arch Pathol Lab Med 2018;142:559-97.

3 Chaturvedi AK, Engels EA, Pfeiffer RM, et al. Human papillomavirus and rising oropharyngeal cancer incidence in the United States. J Clin Oncol 2011;29:4294-301.

4 Mariz BALA, Kowalski LP, William WN, et al. Global prevalence of human papillomavirus-driven oropharyngeal squamous cell carcinoma following the ASCO guidelines: a systematic review and meta-analysis. Crit Rev Oncol Hematol 2020;156:103116.

5 Prabhu SR, Wilson DF. Human papillomavirus and oral disease emerging evidence: a review. Aust Dent J 2013;58:2-10.

6 Rautava J, Syrjänen S. Biology of human papillomavirus infections in head and neck carcinogenesis. Head Neck Pathol 2012;6(Suppl 1):3-15.

7 Fakhry C, Lacchetti C, Rooper LM, et al. Human papillomavirus testing in head and neck carcinomas: ASCO clinical practice guideline endorsement of the College of American pathologists guideline. J Clin Oncol 2018;36:3152-61.

8 Tumban E. A current update on human papillomavirus-associated head and neck cancers. Viruses 2019;11:922.
9 Raubenheimer E, Slavik T, Lemmer L, et al. Human papillomavirus and carcinoma of the mucosal surfaces of the head and neck. $J$ Oral Maxillofac Surg Med Pathol 2018;30:55-9.

10 Syrjänen K, Syrjänen S, Lamberg M, et al. Morphological and immunohistochemical evidence suggesting human papillomavirus (HPV) involvement in oral squamous cell carcinogenesis. Int J Oral Surg 1983;12:418-24.

11 Chaitanya NCSK, Allam NSJ, Gandhi Babu DB, et al. Systematic meta-analysis on association of human papilloma virus and oral cancer. J Cancer Res Ther 2016;12:969.

12 Chi AC, Day TA, Neville BW. Oral cavity and oropharyngeal squamous cell carcinoma--an update. CA Cancer J Clin 2015;65:401-21.

13 Moher D, Liberati A, Tetzlaff J, et al. Preferred reporting items for systematic reviews and meta-analyses: the PRISMA statement. PLoS Med 2009;6:e1000097.

14 Pienaar E, Grobler L, Busgeeth K, et al. Developing a geographic search filter to identify randomised controlled trials in Africa: finding the optimal balance between sensitivity and precision. Health Info Libr J 2011;28:210-5.

15 Ouzzani M, Hammady H, Fedorowicz Z, et al. Rayyan-a web and mobile app for systematic reviews. Syst Rev 2016;5:210.

16 Hoy D, Bain C, Williams $G$, et al. A systematic review of the global prevalence of low back pain. Arthritis Rheum 2012;64:2028-37.

17 Werfalli M, Raubenheimer PJ, Engel M, et al. The effectiveness of peer and community health worker-led self-management support programs for improving diabetes health-related outcomes in adults in low- and-middle-income countries: a systematic review. Syst Rev 2020;9:133.

18 Nyaga VN, Arbyn M, Aerts M. Metaprop: a Stata command to perform meta-analysis of binomial data. Arch Public Health 2014;72:39. 